\title{
Enquête
}

Archives de la revue Enquête

$8 \mid 1993$

Varia

\section{L'envers des Lumières}

Les intellectuels et la culture « populaire » en France (1650-1800)

Jacques Revel

\section{(2) OpenEdition}

Journals

Édition électronique

URL : http://journals.openedition.org/enquete/170

DOI : 10.4000/enquete. 170

ISSN : 1953-809X

Éditeur :

Cercom, Éditions Parenthèses

Édition imprimée

Date de publication : 2 septembre 1993

Pagination : 27-49

Référence électronique

Jacques Revel, «L'envers des Lumières », Enquête [En ligne], 8 | 1993, mis en ligne le 24 février 2006,

consulté le 21 avril 2019. URL : http://journals.openedition.org/enquete/170 ; DOI : 10.4000/

enquete. 170

Ce document a été généré automatiquement le 21 avril 2019. 


\section{L'envers des Lumières}

Les intellectuels et la culture « populaire » en France (1650-1800)

Jacques Revel

1 Sauf exception, nous ne connaissons pas les cultures subalternes ou "populaires » de l'Europe pré-industrielle à travers les témoignages immédiats, spontanés ou au moins autonomes, des acteurs sociaux qui en ont été les protagonistes. Il est devenu banal de le constater: la plupart des informations dont nous pouvons disposer pour les époques anciennes nous sont procurées par des clercs, d'origines, de formations et de compétences souvent fort diverses, mais qui ont en commun de participer aux formes reconnues, légitimes, de la culture d'Ancien Régime. Je choisis de les nommer ici « intellectuels» non que, s'agissant de théologiens, de beaux esprits, de médecins, de voyageurs ou d'administrateurs, une telle qualification soit susceptible de renvoyer à une définition sociale ou socio-professionnelle tant soit peu rigoureuse (pourtant, on le verra, le problème de la professionnalisation de l'expertise n'est pas indifférent à notre propos) ; ni qu'il y ait dans l'ancienne société française, par-delà la diversité des positions et des statuts, une place unifiée, cohérente, clairement identifiable, pour ceux dont la maitrise et la mise en œuvre de savoirs définissent l'activité sociale. Le recours à un tel anachronisme me semble pourtant pouvoir être justifié par une double série de considérations.

Quelle que soit leur origine, les témoins et les commentateurs savants des réalités populaires ont en commun de parler d'un ensemble culturel qui leur est étranger; ils évoquent un monde différent, par rapport auquel ils ont tendance à souligner la cohérence de leur propre culture. Les textes qu'ils nous ont laissés mêlent donc obstinément deux registres qu'il est souvent fort difficile de distinguer : l'observation (dans un sens très large) et l'imaginaire social à l'intérieur duquel ils se situent euxmêmes au moins implicitement. D'autre part, lorsqu'ils font usage d'un savoir pour décrire, expliquer, classer, juger les pratiques populaires, ceux qui nous en ont conservé les traces paraissent avoir en commun le fait de se référer, plus ou moins explicitement, à des formes d'autorité qui les investissent d'une sorte de magistrature sociale et culturelle (notons d'ailleurs que ces autorités peuvent se révéler, à l'occasion, concurrentes voire contradictoires). Une double cohérence s'esquisse ainsi, qui caractérise solidairement 
ceux qui parlent de la culture populaire et leur rapport à ce dont ils parlent. À partir de cette constatation, on voudrait s'interroger ici sur la production d'un certain type de discours et de savoir, et, plus précisément, sur le rapport qui existe entre la position de ceux qui tiennent ce discours et la production d'un objet particulier : le « populaire».

Ce rapport n'est pas donné une fois pour toutes. Bien au contraire, il n'a cessé de se transformer tout au long des trois siècles de la modernité. Ceux qui ont tenté une histoire des relations entre culture savante et culture populaire, ainsi, dans des termes très différents, G. Cocchiara ou R. Muchembled ${ }^{1}$, ont généralement proposé d'en comprendre l'évolution selon un schéma linéaire : de la fin du Moyen-Âge à la naissance de la société industrielle, l'écart ne cesserait de se creuser de l'une à l'autre, et la culture populaire serait l'objet d'une double stratégie de répression et de marginalisation; les folkloristes du XIX ${ }^{e}$ siècle n'en redécouvrent les attraits qu'après qu'elle aura été réduite à l'état de curiosité inoffensive ${ }^{2}$. Dans cette perspective, le rationalisme des Lumières ne fait, à l'évidence, que prendre le relais de l'effort de normalisation des pratiques et des croyances entrepris, depuis le $\mathrm{xVI}^{\mathrm{e}}$ siècle, par les deux Réformes, protestante et catholique, mais aussi bien par la monarchie absolue. Sur une séquence chronologique plus limitée (1650-1800), et en me limitant à l'analyse des formes de l'expertise, je voudrais montrer que cette évolution a peut-être été moins linéaire, plus complexe.

J'en proposerai le schéma suivant que je tenterai d'illustrer dans la suite de cette communication : de part et d'autre de 1750 , date très grossière, on peut identifier deux modèles de traitement du populaire, deux types d'attitudes, de démarches, de discours qui évoluent en sens contraire en construisant des objets opposés. Si nous nous plaçons au milieu $d u x{ }^{e}{ }^{e}$ siècle, ceux qui statuent sur les pratiques populaires sont, très majoritairement, des intellectuels dont le statut d'expert est associé à l'exercice d'une profession : des théologiens, surtout, mais aussi, à l'occasion, des médecins, des juristes, des astronomes; tous se réfèrent à des critères qui sont tout à la fois d'autorité et professionnels. Or, plus on s'avance vers la première moitié du $\mathrm{xVIII}^{\mathrm{e}}$ siècle, plus la compétence de ceux qui parlent du populaire paraît se dissoudre, se dé-professionnaliser, se «dés-autoriser »: se substitue alors à elle une sorte de consensus social et culturel fondé sur le bon sens et sur les convenances - qui sont souvent, d'ailleurs, identifiés. On glisse donc progressivement de l'exercice d'une magistrature qualifiée à la mise en œuvre d'une distinction de nature socioculturelle.

5 C'est un mouvement inverse qui s'esquisse à partir du milieu du XVIII siècle : lentement, de façon multiforme et parfois contradictoire, se recompose un savoir coordonné, articulé, organisé sur les pratiques populaires; les conditions de l'observation qui le fondent sont progressivement codifiées. Des formes d'expertise et de compétence sont redéfinies, qui vont déboucher, à l'extrême fin du siècle, sur l'invention d'une discipline scientifique autonome : l'anthropologie. Son avenir est, d'ailleurs, tout provisoire, mais elle a le temps de préciser son objet, ses procédures de travail, ses règles. En caractérisant plus en détail ces trois figures successives de l'expertise, on voudrait aussi montrer qu'à chaque fois elles proposent du populaire non seulement une approche mais une définition particulière.

6 Convenons d'appeler «populaires » l'ensemble des pratiques qui, dans la culture de la société traditionnelle, n'ont pas de statut légitime. Ces pratiques sont qualifiées (ou, plus exactement, disqualifiées) à travers une série de propriétés qui s'opposent, terme à terme, à celle de la culture établie. 
7 Ces oppositions s'ordonnent selon trois critères principaux, dont les effets sont cumulatifs, mais qui ne se superposent pas exactement. Le premier, le plus étroit et aussi le plus ancien, est un critère de vérité qui oppose un savoir vrai à un savoir faux, ou encore un savoir à un non-savoir ; c'est la position du théologien, par exemple, qui fonde sur ce partage la désignation de ce qui est licite et de ce qui ne l'est pas. Le second, déjà plus lâche, est un critère de rationalité qui oppose des pratiques ou des attitudes cohérentes et explicables (d'un point de vue moral, intellectuel, social, ou encore théologique) à d'autres qui ne le sont pas et qui sont le produit de passions désordonnées et néfastes. Le troisième critère, plus large et plus imprécis encore, peut être dit de « convenance » : il fonde sur la reconnaissance d'un code social plus ou moins explicite la distinction entre ce qui est culturellement acceptable et ce qui ne l'est pas. Entre le milieu du XVII ${ }^{\mathrm{e}}$ siècle et le milieu du XVIII ${ }^{\mathrm{e}}$, ces trois systèmes d'opposition sont simultanément mis en œuvre. Mais la dominante se déplace peu à peu : l'évolution en cours me paraît substituer progressivement au critère le plus rigoureux (vrai/non vrai), le clivage le plus approximatif (ce qui ne signifie nullement le moins opératoire). Vers 1750, on se préoccupe d'abord de reconnaître la civilité des pratiques là où, un siècle plus tôt, on voulait en établir la véracité. Corollairement, bien sûr, l'objet de l'expertise se transforme.

Voici dans la seconde moitié du XVII ${ }^{\mathrm{e}}$ siècle, l'abbé Jean-Baptiste Thiers, le plus célèbre des théologiens français auteurs de traités des superstitions, et qui peut utilement illustrer la première de ces positions. Outre son propre Traité (publié en 1679, très largement amplifié en 1702 et plusieurs fois réédité au cours du XVIII ${ }^{\mathrm{e}}$ siècle), il a consacré de très nombreux libellés, dissertations, mémoires à dénoncer les fausses croyances, les pratiques vaines, l'exercice abusif de la religion. Ce curé de paroisse rigoriste, jansénisant et lié à tout un réseau de prêtres jansénisants, malheureux dans ses rapports avec la hiérarchie catholique, est un homme de la base chez qui la préoccupation pastorale commande l'inlassable militantisme du théologien. Quel que soit le registre dans lequel il se situe, c'est par rapport aux règles du vrai qu'il jauge les conduites qui font l'objet de son analyse. Le Traité des superstitions est tout entier construit sur la confrontation entre une référence théologique (empruntée, pour l'essentiel, à Saint Augustin relayé par Saint Thomas, par les autorités conciliaires et synodales, et qui permet de distinguer la vraie religion de l'idolâtrie) et le monde indéfini des pratiques concrètes ${ }^{3}$. De même, lorsqu'il polémique - entre autres avec Mabillon au tournant des $\mathrm{XVII}^{\mathrm{e}}$ et $\mathrm{XVIII}^{\mathrm{e}}$ siècles - à propos de la véracité des reliques de Saint Firmin d'Amiens (1699) ou de la Sainte Larme de Vendôme (1702), ce pourfendeur de faux cultes mène conjointement une double critique ${ }^{4}$. Thiers vérifie la régularité canonique d'une tradition: la Larme de Vendôme est une croyance, mais une croyance fausse puisque la tradition qui l'atteste « n'est ni divine, ni apostolique, ni épiscopale ou ecclésiastique et qu'il n'y a que ces trois sortes de véritables traditions ». Il critique d'autre part le dossier des preuves avancées par les avocats de la relique au nom d'un bon usage de la connaissance :

«Tous ces événements sont circonstanciés de manière qu'ils pourraient passer pour véritables s'ils n'étaient point destitués de preuves. Mais l'historien qui les raconte n'a pas assez d'autorité pour qu'on le croie sur parole. Il n'a ni style, ni érudition, ni esprit, ni discernement. Il raisonne de travers; il donne aveuglément dans les fables; il n'écrit que par intérêt et par passion; et je n'aurai pas beaucoup de peine à faire voir que, de tous les faits qu'il met en avant, il n'y en a pas un seul qui ne soit fort incertain ou absolument faux... ${ }^{5}$ » 

"discernement m, le " style nou, en sens inverse, la " fable n), la référence à une autorité $\mathrm{du}$ savoir reste dominante et fonde la condamnation, qu'il s'agisse d'une autorité scripturaire ou d'une autorité historique.

Aussi bien la qualification de "populaire» ne renvoie-t-elle chez Thiers à aucune assignation de nature sociale ou socio-culturelle, non plus qu'à aucune qualification intellectuelle. La croyance populaire s'oppose à la croyance catholique en ce qu'elle est particulière et non universelle («chaque royaume, chaque province, chaque diocèse, chaque ville, chaque paroisse a les siennes propres ») et en ce qu'elle est garantie par aucune autorité canonique. La «tradition populaire» (et Thiers est sans doute l'un des premiers auteurs à utiliser cette expression) est, par définition, non recevable car elle identifie abusivement croyance et vérité :

«La croyance de la Larme de Vendôme n'est ni une Tradition divine, ni une Tradition apostolique, ni une Tradition ecclésiastique. Ce n'est tout au plus qu'une tradition populaire qui ne mérite pas de porter le nom de Tradition, n'ayant point la vérité pour fondement ${ }^{6}$. "

11 Entre savoir et non-savoir, la ligne de démarcation ne cesse de se déplacer dans la

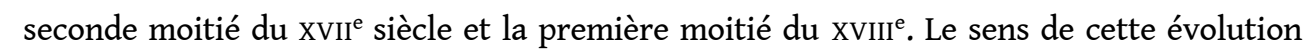
est, en gros, celui d'une laïcisation relative des critères qui fondent le partage. Chez l'Oratorien P. Lebrun, disciple de Malebranche, contemporain de Thiers et qui publie en 1702 une Histoire critique des pratiques superstitieuses qui ont séduit les peuples et embarrassé les savants, les faits de superstitions sont passés, comme l'indique le titre de son livre, au double crible de la science et de la foi. Les procédures de l'expertise se veulent d'abord méthodiques et scientifiques - comme elles le seront, plus explicitement encore, dans l' Histoire des oracles de Fontenelle, quelques années plus tard. Mais ici encore, l'évolution en cours n'est ni simple, ni linéaire. Elle ne se réduit pas à une laïcisation imparable qui vouerait à la déqualification, voire au déclassement, les clercs-experts. Et ce pour une double raison. D'abord parce que les clercs eux-mêmes adoptent au moins partiellement les nouveaux critères et sauvent ainsi, en partie au moins, leur prétention à la magistrature culturelle. C'est l'attitude du rationalisme catholique. Ensuite parce que, même lorsqu'il est militant, le rationalisme se montre attentif à ne point trop empiéter sur le territoire des théologiens. De la première attitude témoigne, en plein cœur des lumières, l'entreprise d'un Dom Calmet ${ }^{7}$. Chez ce dominicain, victime célèbre des sarcasmes de Voltaire et qui publie en 1746 une Dissertation sur les apparitions des anges, des démons et des esprits et sur les revenants et vampires de Hongrie (plusieurs fois rééditée), on est sensible à l'inlassable volonté - parfois un peu maladroite - de ménager foi et raison en distinguant un surnaturel "réel» (c'est-à-dire raisonnable) et l'ensemble des superstitions populaires qui sont le fait de l'ignorance et qu'il n'hésite pas à charger :

«On remarque que plus l'ignorance est grande dans un pays, plus aussi la superstition y règne, et que l'esprit de ténèbres y exerce un plus grand empire à proportion de ce que les peuples sont profondément plongés dans l'erreur et le désordre ${ }^{8}$."

12 Il n'y a plus guère de place dans cette analyse pour le démon qui obsédait Thiers. Mais voici, à l'inverse, les prudences de la raison`. On a rappelé récemment que jusque dans ce bastion du militantisme éclairé qu'est l'Encyclopédie, les articles qui traitent des diverses formes de la superstition observent une sage discrétion (Goulemot, 1980). Rédigés pour la plupart par le chevalier de Jaucourt, ils se réfèrent volontiers aux théologiens les plus traditionnels (à Thiers en particulier) et invoquent plus volontiers l'usage de la bonne

Enquête, 8 | 1993 
raison que les exigences du rationalisme laïque. Ils s'en remettent, en gros, aux théologiens pour déterminer les limites que ne saurait franchir l'exercice de la raison. La définition même de la superstition semble indiquer la voie d'une sorte de partage des taches : « culte de religion, faux, mal dirigé, plein de vaines terreurs, contraire à la raison et aux saines idées qu'on doit avoir de l'Être suprême ». Lorsque l'on aborde le sortilège, les choses paraissent plus claires encore (c'est-à-dire plus estompées) :

«Le sortilège est compris dans ce qu'on appelle magie; mais il a particulièrement pour objet de nuire aux hommes soit en leur personne, soit en leurs bestiaux, plantes et fruits de la terre. Il n'appartient qu'aux théologiens de traiter une matière si délicate ».

Prudence, sans doute; mais aussi conviction qu'il existe une solidarité des détenteurs d'un bon usage de la raison face à ceux qui ne s'en servent pas ou mal. Voici qui nous introduit à une seconde ligne de clivage, identifiable, en fait, bien avant l'âge de l'Encyclopédie.

la première moitié du XVII ${ }^{e}$ siècle, en effet, un autre type de partage s'est esquissé entre la culture des élites et le fond des pratiques populaires. Là où le $\mathrm{XVI}^{\mathrm{e}}$ siècle avait connu des formes d'intérêt, d'ailleurs très diverses, pour les productions des cultures subalternes, et, en particulier pour leurs expressions régionales, pour les langues, pour les proverbes assidûment collectionnés et compilés ${ }^{10}$, l'âge classique instaure une distance aisément repérable. Sans doute continue-t-on à publier, en moins grand nombre cependant, des recueils de proverbes; sans doute encore le trésor des contes populaires fait-il son entrée, ambiguë, dans la haute littérature avec les Contes de ma Mère L'oye de Charles Perrault (1697). Mais, qu'il s'agisse de permanences ou de retrouvailles, ces jalons n'indiquent pas la ligne de pente majeure du siècle. Après René Pintard ${ }^{11}$, J.-M. Goulemot a justement insisté sur le rôle déterminant qu'a joué dans cette évolution le milieu des libertins érudits. Or ceux-ci, même s'ils constituent un groupe minoritaire et, somme toute, marginal, revendiquent un type de contrôle social et culturel fort différent de celui que nous venons d'envisager. Leur prétention à exercer un magistère n'est plus fondée sur l'appartenance à une profession qui les instituerait détenteurs d'un savoir opératoire parce qu'autorisé. Ils participent d'ailleurs de réseaux beaucoup plus diffus et divers que les solidarités qui unissaient, par exemple, les théologiens correspondants de l'abbé Thiers. Ces esprits forts ne se recrutent, ni ne se reconnaissent sur la base d'une compétence normative ; ils ne prétendent pas détenir une vérité, mais ne veulent avoir en commun qu'un usage libre et salutaire de la raison critique.

Or ce bon usage de la raison n'est en rien la chose au monde la mieux partagée. Sans doute les voies de la raison sont-elles, par définition, accessibles à tous, mais l'usage en reste un privilège inégalement distribué. Il est le signe de reconnaissance d'une élite inédite qui veut s'ériger en aristocratie nouvelle. Au moment même où s'élabore la raison d'État, elle attend de la monarchie absolue qu'elle reconnaisse et qu'elle confirme ses talents et ses privilèges, et qu'elle fasse de l'État l'instrument d'une socialisation efficace de la bonne raison. Un modèle des rapports entre le savant et le politique est ainsi proposé très tôt, que le xviII ${ }^{\mathrm{e}}$ siècle saura retrouver et amplifier. Il convient, ici encore, de ne point simplifier à l'excès. Le combat pour la raison est mené sur plusieurs fronts simultanément pendant le $\mathrm{XVII}^{\mathrm{e}}$ et la première moitié $\mathrm{du} \mathrm{XVIII}^{\mathrm{e}}$ siècle. Les grands débats sur le croyable, autour de la réalité des faits de sorcellerie et de possession dans les années 1630-1650, autour de la nature et de la signification des comètes dans les années 1650-1680, mettent aux prises les libertins et l'«ignorance ", mais aussi les clercs et les 
laïcs, les «scientifiques » et les amateurs, les différents corps professionnels entre eux, selon des stratégies et des solidarités mouvantes et infiniment complexes ${ }^{12}$ : on voit ainsi les clercs, menacés à la fois par la persistance des croyances communes, par la dénonciation des libertins et par la compétence des savoirs techniques (ceux des médecins ou ceux des astronomes, par exemple), rejoindre par étapes, avec un mélange de réticence et d'empressement, le camp de la raison positive.

Il reste que, dans tous les cas, la dénonciation de l'erreur ou de la fausse croyance s'accompagne désormais d'une disqualification sociale. Le populaire n'est plus, comme dans la figure précédente, l'indice d'une non-conformité épistémologique, il est producteur d'obscurité et d'erreurs, c'est ce qu'exprime en clair l'intendant des fortifications, Petit, dans une Dissertation sur la nature des comètes (1654), d'ailleurs commanditée par la monarchie pour donner la leçon raisonnable d'un événement astronomique qui bouleverse l'Europe crédule : «Toute l'Europe fut consternée de peur, je parle du peuple ignorant, jusqu'à croire à la fin du monde...13 ». Trente ans plus tard, lors du passage de la comète de 1680 , on parle, plus explicitement encore, de la fausse croyance comme d'une " maladie populaire ", comme de la trace fossilisée d'un archaïsme social et culturel dont le peuple est, par position, porteur, et qui constitue, tout à la fois, l'indice et la justification de sa nécessaire sujétion. Les pratiques populaires témoignent donc d'un âge révolu, elles ne sont plus que le conservatoire des idées fausses de l'humanité, de l'enfance des hommes (le thème est ici promis à une opiniâtre fortune). C'est ce que dit en clair le Journal des Savants en esquissant une trajectoire saisissante, qui voit l'astronomie aristotélicienne rejoindre l'enfer des croyances populaires :

« L'ancien philosophe l'a crû, parce que comme elle vouloit que les comètes fussent sublunaires et que leur nature ne fût qu'un amas d'exhalaisons de la terre, quand il arrivoit que ces exhalaisons prenoient feu, ce qui ne pouvoit que marquer une grande intempérie de la Région Élémentaire, il devoit s'ensuivre, suivant cette opinion, quelque grande et considérable révolution. Mais depuis que l'on a sçu que les comètes étoient des corps célestes, on s'est désabusé de cette erreur qui n'est plus qu'une erreur populaire ${ }^{14}$. "

On voit donc comment s'opère l'insensible glissement d'une dénonciation faite au nom de la raison commune ou de la connaissance scientifique à des formes de disqualification fondées sur une assignation sociale dévalorisante. On aborde ainsi une troisième ligne de partage.

18 Je ne fais qu'évoquer ici la réorganisation de l'espace culturel qui caractérise l'âge classique, en gros du milieu du $\mathrm{XVII}^{\mathrm{e}}$ au milieu du XVIII ${ }^{\mathrm{e}}$ siècle et qui est un phénomène trop connu pour qu'il soit besoin de s'y arrêter longtemps. Appuyée sur l'état monarchique et sur les institutions nouvelles qu'il met en place ainsi que sur les entreprises de l'Église post-tridentine, une normalisation culturelle multiforme est à l'ordre du jour. On en retrouve partout sensibles les effets: dans la codification des œuvres et des critères du goût, dans la re-formulation des règles de conduite sociale et des formes licites de sociabilité, dans les prescriptions d'une morale collective. Toute une gamme de modèles élitaires, généralement urbains, de comportements et de valeurs sont désormais proposés, et opposés à des formes populaires supposées, la plupart du temps paysannes. Les pratiques religieuses font alors l'objet d'un intense travail d'épuration et de contrôle qui est, pour l'essentiel, assuré par des experts professionnels, comme on l'a vu déjà. Mais le succès même de leurs entreprises, la vogue de leurs ouvrages bientôt promus au rang de recueils de curiosa et continûment réédités pour les amateurs du XVIII ${ }^{\mathrm{e}}$ siècle, font bien voir que tant la discrimination ecclésiastique à fondement théologique 
que les prétentions de la raison sont désormais redoublées et relayées dans un plus large public d'honnêtes gens par un ostracisme social ; on en trouvera cent témoignages dans la libre correspondance de Mme de Sévigné, par exemple. Au même moment, les fonctionnements et les usages de la langue font eux aussi l'objet d'un double travail d'inventaire et d'analyse scientifique d'une part, de codification sociale de l'autre. Des institutions publiques comme l'Académie française, des techniciens de la grammaire et de la logique, des compilateurs de dictionnaires arrêtent les règles du bon usage de la langue qui est aussi un usage privilégié et exclusif d'autres usages (bourgeois, populaires, régionaux, professionnels, techniques, etc.) qui se voient disqualifiés. À la civilité des pratiques légitimes s'oppose désormais la marginalité des usages non réglés qui sont aussi ceux du plus grand nombre. La norme est devenue classificatoire. Car si le populaire évoque une assignation sociale péjorative, il ne renvoie en fait à aucune identité sociale précise : on y retrouve sans doute les superstitions de la campagne et l'argot de la rue parisienne, mais aussi le fonds déchu de l'ancienne littérature "cultivée » que relève alors la Bibliothèque bleue, les balourdises du Bourgeois Gentilhomme et la "vieille chanson ", œuvre de "nos pères tous grossiers ", qu'Alceste prise tant contre le goût du jour. Le populaire est maintenant le monde négatif des pratiques non licites, des conduites curieuses ou erratiques, de l'expressivité sans contrôle, une nature en face d'une culture - celle-là même que croiront découvrir au siècle des Lumières les inventeurs de la France profonde.

On constate donc l'existence et la mise en œuvre simultanées d'une série de critères qui ont pour caractéristique d'être d'autant plus englobants qu'ils sont plus lâches. Or, entre 1650 et 1750, ce sont les moins rigoureux, donc les plus exclusifs qui, peu à peu, prévalent. Ces critères sont, pour une part, maniés par des groupes dont la compétence et l'expertise sont d'autant plus strictement définies que leurs principes de référence sont plus précisément explicités. Des démonologues, comme Bodin, des théologiens comme Thiers ou Lebrun, des médecins, des astronomes, sont des professionnels d'un savoir; ils maîtrisent des techniques particulières et en usent à l'intérieur d'un champ d'application clairement délimité. Or leur rôle ne cesse de reculer entre XVII et $\mathrm{XVIII}^{\mathrm{e}}$ siècle, tandis que passe au premier plan l'affirmation d'un code social et culturel de reconnaissance et d'exclusion. Ce code est lui-même d'autant plus efficace socialement qu'il n'explicite pas ses règles de fonctionnement ; il se pare des prestiges de l'évidence : il se donne en effet pour une collection d'usages communs, mais dont la maitrise effective demeure réservée à un petit nombre. Il n'est pas l'affaire de spécialistes, mais il est approprié par ceux qui, en en disposant, se qualifient à leurs propres yeux et contre les outsiders. Là où existait un corps de savoir, si arbitraire soit-il jugé, est désormais proposé un système de connivences.

Ces déterminations ont d'évidentes conséquences sur la constitution même de l'objet visé, le «populaire ». Comme la plupart des autres théologiens de la superstition, J.-B. Thiers reste écartelé, dans son Traité, entre la rigueur d'une définition purement normative (religion vs superstition) et l'inventaire empirique, indéfini, des figures concrètes du mal qu'il prétend poursuivre, de ces pratiques illicites qu'il faut inlassablement identifier, décrire, classer. C'est sans doute à cause de cette contradiction non résolue que le geste de classer prend chez lui une telle importance. Il est au fond bien plus important que l'examen des principes d'une classification qu'on ne saurait remettre en cause (puisqu'elle est d'institution divine et ecclésiastique) ou encore que la collecte concrète de l'information. Assez curieusement, on ne trouve guère chez cet homme de terrain, que 
sa correspondance conservée nous fait voir échangeant avec d'autres hommes de terrains fiches et références bibliographiques, le souci de vérifier sur place la qualité des informateurs ou la validité des informations; et jamais on ne rencontre l'idée que les pratiques qu'il recense pourraient avoir entre elles une cohérence porteuse de signification. Un témoignage posthume évoque bien, d'ailleurs, sa manière de travailler :

«Quand il s'était mis en tête d'écrire sur un sujet qu'il choisissait toujours extraordinaire ou bizarre, il feuilletait parmi toutes les tables de ses livres, dont il avait bon nombre d'assez singuliers, et allait à la quête dans les bonnes bibliothèques ou chez ses amis, pour fournir à ses desseins et jetait sur le papier ses découvertes ${ }^{15}$. »

Il serait vain, sans doute, de s'étonner de l'absence de préoccupations « ethnographiques » chez un auteur dont ce n'est en rien là le projet. Son métier et la compétence dont il est armé le mettent sans doute au contact quotidien des pratiques populaires ; mais ce qui le meut est la défense et l'illustration d'une norme, vrai sujet de cette œuvre immense, et dont la fécondité de l'activité classificatoire suffit, aux yeux du clerc, à prouver l'efficacité concrète.

Encore Thiers pense-t-il qu'il existe un vrai et un faux, et des règles pour les séparer. Son objet n'est pas le populaire mais la vraie foi qu'il doit garder et imposer. Si nous nous plaçons à l'autre bout de la chaîne, là où le discours de l'exclusion sociale se fait le plus en général et le moins explicite, il change de statut et de nature. Il apparaît à la limite sans auteur et sans objet, ce qui ne veut pas dire sans efficacité sociale, bien au contraire. Sans auteur : il est tenu anonymement par une collectivité qui se reconnaît à travers lui, mais indépendamment de toute capacité technique d'expertise; c'est un discours que le groupe tient sur lui-même et non la mise en œuvre ou l'illustration d'une norme ou d'une compétence. Sans autre objet que le geste de qualifier ou de disqualifier en fonction d'une position sociale. Là où Thiers s'épuisait à construire des taxinomies indéfinies, là où Lebrun cherchait à éprouver des règles sûres pour séparer le vrai du faux, l'abbé Langlet Dufresnoy propose, en 1751, un Recueil de dissertations anciennes et nouvelles sur les apparitions, les visions et les songes, qui compile (utilement, d'ailleurs) des « histoires extraordinaires » dont il se flatte « qu'on ne m'accusera pas de croire tout ce que j'ai fait imprimer ici $^{16}$. » Il prend ses distances par rapport à un corpus de texte qu'il choisit de ranger dans l'ordre le plus neutre possible, l'ordre chronologique, et qu'il livre à la curiosité de ses lecteurs, sans suggérer ni jugement, ni règle de lecture. Dans son propos, il n'y a plus de place pour l'expertise dont le besoin est, apparemment, plus ressenti puisqu'on se situe désormais dans l'ordre de l'évidence, virtuellement partagée, pratiquement discriminante.

Vers la moitié du XVIII ${ }^{\mathrm{e}}$ siècle, au moment même où paraît achever de se dissoudre la figure ancienne de l'expertise sur le populaire, un mouvement de sens contraire semble s'amorcer. Avant la fin du siècle, en l'espace de deux générations, il aboutira à la redéfinition d'un objet d'observation (les pratiques populaires), à de nouvelles formes de professionnalisation fondées tout à la fois sur l'accumulation d'un capital de connaissances et sur la codification nouvelle de l'observation.

Face aux textes que nous venons d'évoquer, et dont la vocation était de distinguer, en les prohibant ou en les disqualifiant, les productions culturelles illicites ou illégitimes des formes autorisées ou dominantes de la culture, une autre série d'énoncés propose un changement d'attitude décisif. On peut, très approximativement, les caractériser comme " pré-ethnographiques » non pas dans la mesure où ils seraient porteurs des bribes d'une 
information de type ethnographique (car c'est le cas aussi des traités des superstitions du $\mathrm{XVII}^{\mathrm{e}}$ siècle, des recueils de curiosités du XVIII ${ }^{\mathrm{e}}$, et de tant d'autres documents avec eux et parfois bien avant eux), mais en raison de l'attitude qu'ils adoptent en face de l'objet dont ils proposent de rendre compte. Ils sont sans doute fort divers : voyages à l'intérieur des provinces, documents statistiques, rapports administratifs, monographies descriptives se multiplient après 1750 , et sont relayés par les grandes enquêtes du dernier tiers du siècle. Ces textes ont pourtant en commun un certain nombre de caractères. À des degrés variables, ils manifestent la découverte et la reconnaissance d'un niveau culturel différent, étrange ou étranger (ou, comme on le dit souvent alors : curieux). Mais la curiosité ne se fonde plus, cette fois, sur une relation d'extériorité ; elle rend possible au contraire un type d'interrogation inédit. Car la reconnaissance d'une culture autre comme telle n'est possible, et c'est là que se marque la nouveauté, que parce qu'il existe entre « eux » et "nous », entre l'observateur et le sujet de l'observation, une très forte conscience d'identité qui passe par l'affirmation explicite d'une unité fondamentale des formes de la vie des hommes. La description et la compréhension des figures de l'autre doivent ainsi être restituées dans une histoire des origines collectives de l'humanité.

On pense bien sûr aux grands théoriciens du siècle, à Rousseau, mais aussi à Buffon ${ }^{17}$. Mais il convient d'être attentif surtout à la masse des tentatives, souvent bien plus modestes et bricolées, faites pour reconstituer par séquences et sur pièces, la généalogie sociale et culturelle des hommes; à tous ces efforts pour retrouver les soubassements anciens qui garantiront la cohérence de leurs productions et de leur histoire. Car chaque " curiosité ", chaque épave, chaque bribe retrouvée des pratiques populaires est comme l'indice d'une couche archaïque et le témoignage d'un passé enfoui. L'archaïsme a changé de sens : il n'est plus la marque d'un âge révolu et déclassé ; il devient le vestige précieux d'un enchaînement dont la compréhension est nécessaire à l'intelligence de la société présente. La collecte des faits est sans doute, et pour longtemps encore, celle de l'insignifiant mais elle n'a de sens que parce que ces faits sont appelés à devenir signifiants à l'intérieur d'un ordre que le siècle se donne pour tâche de retrouver, de reconstruire et de comprendre.

Le populaire conserve désormais la mémoire des âges passés. Il doit être compris comme un produit social lié à des conditions de production particulières, et non plus comme le résultat négatif d'une disqualification. Destutt de Tracy l'exprime bien dans ses Éléments d'idéologie, au terme même de ce mouvement (1801-1804) :

« Les sauvages nous donnent souvent lieu d'admirer que des hommes si peu éclairés fassent des combinaisons si fines et que, les faisant, ils soient tout à fait incapables d'en faire d'autres qui nous paraissent moins difficiles. Dans les sociétés civilisées, la classe qui a les communications les moins étendues et les moins variées offre des phénomènes analogues. Le paysan des campagnes écartées, ceux des montagnes, sont remarquables par la rectitude d'un petit nombre de combinaisons, l'ignorance absolue d'une foule d'autres et leur incapacité à en faire de nouvelles ${ }^{18}$.»

Texte remarquable à un double titre : parce que, au-delà d'une opposition simpliste entre le sauvage et le civilisé, il suggère de relativiser l'ordre et la hiérarchie des productions culturelles; parce que, d'autre part, il ne propose pas de penser une civilisation contre l'autre, mais souligne l'importance des statuts sociaux à l'intérieur de chaque civilisation. L'identification du paysan au sauvage, vrai lieu commun de l'Europe éclairée, ne me paraît pas introduire ici de jugement de valeur dépréciatif, mais renvoyer plutôt à une hiérarchie des formes de la vie sociale intelligible à l'intérieur d'une histoire dont on sait bien qu'elle fait, au XVIII ${ }^{e}$ siècle, l'objet de valorisations pour le moins contradictoires. 

extraordinairement divers de recherches "génétiques" menées depuis trente ou quarante ans. Voici, par exemple, Antoine Court de Gébelin parti en quête de l'Origine du langage et de l'écriture dans un monde primitif analysé et comparé avec le monde moderne, considéré dans l'histoire naturelle de la parole (1775). Partant du postulat que l'on peut « ramener toutes les langues à une seule langue dont elles ne sont que des nuances ", il veut identifier la langue primitive de l'humanité, puis, en aval, celle de l'Europe (le celtique, « la langue... qui a servi de base à nos origines françaises ») et retracer l'histoire de ses progressives différenciations. Le sens de la démarche scientifique est clair: exorciser le "fantôme effrayant de la multitude des langues " - qui est aussi celui de l'hétérogénéité du social - c'est aussi rendre historiquement compréhensible la logique de leurs différences. Presque contemporaine, l'analyse de Legrand d'Aussy dans l'Histoire de la vie quotidienne des français depuis l'origine de la nation jusques à nos jours (1782) est inspirée par une préoccupation comparable. Lorsque l'auteur traite, de façon très neuve, de la diversité des pratiques alimentaires dans l'espace français, avec un constant souci de le localiser et de les dater, c'est qu'il veut comprendre la multiplicité à l'intérieur d'une perspective génétique, parce qu'elle renvoie, comme son titre l'indique, à une histoire. largement à faire) peut être comprise comme manifestant la volonté de construire dans l'histoire les fondements d'une anthropologie. Rien n'en témoigne mieux, tout au début du XIX ${ }^{e}$ siècle, que les travaux de l'Académie celtique, et, en particulier, la préparation du célèbre questionnaire de Dulaure et Mangourit (1807), étudiée par Mona Ozouf ${ }^{19}$. Dans le réseau serré des 51 questions retenues, les académiciens entendent retenir de façon aussi systématique que possible ce qui reste repérable de la culture traditionnelle des Français. On a justement souligné que la collecte était, dans son principe, indéfinie; que le questionnaire demeurait ouvert, et qu'il définissait d'ailleurs «moins un objet qu'un espace à baliser ». Mais c'est que l'espace social de la culture est sans doute le vrai objet de l'enquête. L'entreprise part de deux postulats qui en commandent la logique : elle veut fonder culturellement l'unité de la nation française (que l'on s'efforce, au même moment, de construire politiquement); elle affirme en second lieu que chaque détail collecté, chaque « survivance » observée doit trouver sa place dans un système culturel cohérent qu'il importe de recomposer car il est la preuve même de l'unité recherchée.

On voit bien en quoi la perspective s'est renversée, en quoi le rapport des intellectuels au populaire a changé. Ce qui paraît résister à l'assimilation culturelle est désormais ce qui devient l'objet principal de l'intérêt et de l'étude. Le voyageur cultivé du XvII ${ }^{e}$ siècle, et encore dans la première moitié $d u \mathrm{xVIII}^{\mathrm{e}}$, que visitait-il ? Des villes, des monuments témoins d'une histoire déjà constituée (la "grande histoire »); il s'initiait aux œuvres d'art, découvrait les pratiques culturelles dominantes, apprenait à reconnaître les formes de la sociabilité des élites. Son homologue de la fin du XVIII ${ }^{\mathrm{e}}$ siècle suit de tout autre parcours. C'est vers 1770-1780, selon M. Vovelle, que les curiosités des visiteurs de la Provence s'élargissent et qu'ils commencent d'explorer plus systématiquement l'intérieur de leur province. L'intérêt du voyageur le porte désormais là où il n'y a ni monument explicite, ni histoire repérable, ni pratiques immédiatement reconnaissables: vers les écarts, les bouts-du-monde, les montagnes ou les îles (que l'on pense à la vogue de la Bretagne, des Pyrénées ou des Alpes!). Cet exotisme de l'intérieur est tout à la fois un retour aux origines et une exploration du social. Aux beaux temps de l'Idéologie, De Gérando en donnera une sorte de justification théorique parfaitement explicite : 
«Le voyageur philosophe qui navigue vers les extrémités de la terre, traverse la suite des âges; il voyage dans le passé ; chaque pas qu'il fait est un siècle qu'il franchit. Ces îles inconnues auxquelles il atteint sont pour lui le berceau de la société humaine ${ }^{20}$. »

31 On voit aussi à quel point le projet culturel est, en profondeur, associé à un projet politique. La chose devient patente au temps de la Révolution et de l'Empire, mais le lien est bien plus ancien dans le $\mathrm{XVIII}^{\mathrm{e}}$ siècle. Une bonne part de la littérature qui découvre les formes de l'existence populaire est alors le fait d'administrateurs, de médecins, d'économistes. Sans doute n'inventent-ils pas, eux non plus, le genre de la description administrative attesté en France au moins depuis le XVII siècle, ailleurs parfois plus tôt encore. Mais leurs textes, qui sont d'ailleurs incomparablement plus nombreux qu'ils ne l'étaient jusqu'alors, innovent aussi par leur volonté manifeste d'intervention sociale et d'efficacité pratique. La plupart d'entre eux laissent paraître une question centrale: comment rendre possible une meilleure gestion de la société, comment frayer les voies de l'innovation? Pourtant la volonté d'innovation se heurte souvent aux résistances de la tradition : des plaintes répétées de la littérature physiocratique sur l'inertie des habitudes paysannes aux enquêtes de François de Neufchâteau sur l'esprit public des provinces pendant le Directoire, en passant par les inquiétudes de Grégoire sur la pénétration de la conviction révolutionnaire dans les campagnes aux premières années de la Révolution et par les correspondants de la Société royale de médecine, les gestionnaires des Lumières découvrent l'obstacle des comportements et des mentalités populaires. Ici encore, ils ne sont pas les premiers, assurément, à identifier la France rurale, lieu emblématique qui symbolise commodément à leurs yeux l'opacité sociale et la tradition culturelle, comme le monde qui résiste à leurs entreprises. Mais les choses ont changé. Ils sont, d'une part, bien plus soucieux d'efficacité empirique que ne l'étaient leurs devanciers : il suffit, pour s'en rendre compte, de comparer l'enquête sur l'état du Royaume menée en 1697 par le duc de Beauvilliers pour l'instruction du dauphin, et les enquêtes administratives ou les topographies médicales de la seconde moitié du XVIII ${ }^{e}$ siècle. L'efficacité gestionnaire est donc requise de se doubler d'une pédagogie à destination populaire.

Elle suppose, en outre, que l'on ait déjà repéré et expliqué ce qui dans le peuple détermine le refus obstiné du progrès, et donc qu'au lieu de considérer les comportements comme les témoins singuliers et erratiques d'une infériorité de statut, on cherche à en comprendre la cohérence : ce qui fait système et qui, dans l'esprit de ces «primitifs » de l'ethnographie, permet d'associer dans un même tableau explicatif des déterminations géographiques (sols, climats...), les formes de l'implantation humaine, des règles de sociabilité, une physiologie particulière, des traits psychologiques constants, caractères dont l'interaction doit, en dernière analyse, rendre compte du retard des esprits et du blocage face à l'innovation. Le questionnaire, que rédige en 1790 l'abbé Grégoire pour préparer les mesures qui devront, dans son esprit, permettre l'universalisation du français et la réduction des langues régionales, est tout à fait représentatif de cette démarche nouvelle. Enfin, à ces évolutions déjà marquées, la Révolution vient ajouter une rupture plus expressément politique encore. Car ce peuple qui demeure à la traîne des Lumières, voici qu'il est aussi l'acteur proclamé de la Révolution, désormais en charge du destin de la nation. Il n'est plus seulement placé aux origines de toute humanité, il en est aussi l'avenir. Au moment même où ses réticences à se rallier au nouveau cours posent le plus de problèmes aux politiques, il fait l'objet de la plus forte valorisation idéologique. D'où l'urgence de le comprendre, puisque c'est par cette compréhension que passe nécessairement son incorporation au projet collectif ${ }^{21}$. 
Culturel, politique, il va de soi que la distinction entre deux registres de préoccupations aussi intimement associés est ici fort artificielle: les deux motivations s'enchevêtrent, parfois à l'intérieur d'une phrase. Mais il importe de souligner, en terminant, qu'à la nouvelle figure $d u$ peuple qui naît des Lumières et $d u$ volontarisme révolutionnaire correspond une fois encore un nouveau statut de l'observateur et une forme inédite de l'expertise. Sans doute, l'évolution s'inscrit-elle parfois à l'intérieur de formes institutionnelles traditionnelles (le réseau académique, par exemple) ou de genres littéraires anciens (ainsi le voyage littéraire); mais ce n'est pas le cas le plus fréquent. Les topographies et les dictionnaires médicaux, les descriptions statistiques, les rapports administratifs, l'enquête surtout qui se multiplie dans les trente dernières années de l'ancien Régime et plus encore pendant la Révolution, sont les formes privilégiées du nouvel intérêt pour le populaire. On y rassemble une information souvent bien plus austère que ne l'était la discussion philosophique, mais avec l'intention explicite d'accumuler dans ces publications les éléments d'un savoir positif sur le peuple.

Des institutions neuves surgissent aussi dont l'organisation fait bien voir le projet scientifique qui se définit alors. Déjà les formes textuelles nouvelles suggéraient bien souvent un effacement de l'auteur derrière la présentation d'un savoir communicable et capitalisable : chaque statistique peut ainsi être considérée comme une pierre apportée à l'enquête collective qui fera connaître l'espace français selon des règles de description en gros standardisées. En outre, à ces entreprises, les sociétés de pensée révolutionnaires (par exemple la Société des Amis de la Constitution, qui fournit tant de correspondants à Grégoire), et surtout des organisations spécialisées donnent à la fois une forme de sociabilité savante et un projet scientifique clairement avoué. On a déjà évoqué la plus célèbre d'entre elles, l'Académie celtique. Plus significative encore est peut-être sa devancière, la Société des Observateurs de l'Homme (1799-1805) ${ }^{22}$. Cette dernière se définit elle-même comme un groupe savant, agissant collectivement, et rassemblant des chercheurs venus d'horizons complètement différents - on y trouve des médecins et des géographes, des linguistes et des naturalistes, des historiens et des philosophes, des publicistes et des voyageurs - autour d'un même projet scientifique, l'« étude de l'homme physique, intellectuel et moral». Ses cinquante membres mettent en commun leurs compétences spécialisées pour tenter d'élaborer, tout à la fois, la théorie de leur recherche (c'est l'idéologie qui vaut leur nom générique aux membres de la secte) et d'en codifier la pratique.

35 Car autour de leur objet, l'homme aussi largement défini, les Idéologues proposent une réorganisation disciplinaire, un contrôle de l'exercice professionnel des observations savantes, et même une pédagogie de la recherche. Uniformiser, codifier, professionnaliser, systématiser l'étude du populaire, c'est-à-dire constituer le champ et les critères d'une expertise : pour la première fois sans doute les pratiques populaires font l'objet d'un projet de traitement cohérent au sein d'une première élaboration des sciences de l'homme, et ne sont plus jugées à l'aune de critères empruntés à des espaces culturels et sociaux étranger ou concurrents. Pourtant, on le sait, le projet n'a pas d'avenir immédiat en France. Les Idéologues seront bientôt dispersés et le savoir sur le peuple à nouveau éclaté, pour l'essentiel entre une statistique morale souvent normative et le folklorisme romantique qui naît dans le premier tiers du XIX ${ }^{e}$ siècle. 


\section{NOTES}

1. G. Cocchiara, Storia del folklore in Europa, Turin, Paolo Boringhieri, 1971;

R. Muchembled, Culture populaire et culture des élites dans la France moderne ( $\mathrm{XV}{ }^{e}-\mathrm{XVIII}{ }^{e}$ siècles) , Paris, Flammarion, 1978.

2. M. de Certeau, D. Julia. J. Revel, « La beauté du mort : le concept de culture populaire », Politique aujourd'hui, déc. 1970, p. 3-23.

3. R. Chartier, J. Revel, « Le paysan, l'ours et Saint Augustin », in La découverte de la France au $17^{\mathrm{e}}$ siècle, Actes du colloque de Marseille, Centre Méridional de Rencontres sur le $17^{\mathrm{e}}$ siècle, 25-28 janv. 1979, Paris, Éd. du CNRS, 1980, p. 259-264.

4. J.-B. Thiers, Dissertation sur la Sainte Larme de Vendôme, Paris, 1699 ; Dissertation sur le lieu où repose présentement le corps de Saint Firmin le Confês, III Evesque d'Amiens, Paris, 1699.

5. Dissertation sur la Sainte Larme, Amsterdam, 1751, p. 125.

6. lbid., p. xxxix.

7. B. Kôpeczi, «Un scandale des Lumières : les Vampires », in R. Trousson, ed., Thèmes et figures du siècle des Lumières. Mélanges offerts à Roland Mortier, Genève, Droz, 1980,

p. 123-135.

8. Dom Calmet, Dissertation sur les apparitions, p. 178.

9. Ce qui suit se fonde sur l'étude de J.-M. Goulemot, « Démons, merveilles et philosophie à l'âge classique ». Annales ESC, 6, 1980, p. 1223-1250.

10. N. Z. Davis, Society and Culture in Early Modern France, Stanford (CA), Stanford University Press, 1975, chap. 8, « Proverbial Wisdom and Popular Errors », p. 227-267.

11. R. Pintard, Le libertinage érudit dans la première moitié du XVII siècle, Paris, Boivin, 1943.

12. R. Mandrou, Magistrats et sorciers en France au XVII ${ }^{e}$ siècle. Une analyse de psychologie historique, Paris, Plon, 1968 ; E. Labrousse, L'entrée de Saturne au Lion (l'éclipse de soleil du 12 août 1654), La Haye, Martinus Nijhoff, 1974.

13. Cité par J.-M. Goulemot, « Démons, merveilles et philosophie à l'âge classique », p. 1226-1227. [C'est moi qui souligne.]

14. Ibid., p. 1227. [C'est moi qui souligne.]

15. Bibliothèque municipale, Grenoble, Ms. 227, Recueil de lettres de D. Bonaventure d'Argonne, ff. 28-29.

16. N. Lenglet Dufresnoy, Recueil de dissertations anciennes et nouvelles sur les apparitions, les visions et les songes, avertissement, Avignon \& Paris, J. N. Leloup, 1751 ; J.-M. Goulemot, «Démons, merveilles et philosophie à l'âge classique », p. 1234.

17. M. Duchet, Anthropologie et histoire au siècle des Lumières, Paris, Maspero, 1971.

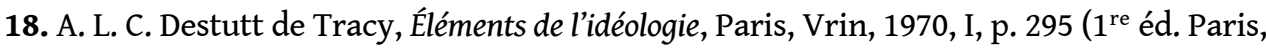
1801).

19. M. Ozouf, « L'invention de l'ethnographie française. Le questionnaire de l'Académie celtique ", Annales ESC, 2, 1981, p. 210-230.

20. J.-M. de Gérando, Considérations sur les diverses méthodes à suivre dans l'exploration des peuples sauvages, Paris, 1800 ; rééd. in J. Copans et J. Jamin, Aux origines de l'anthropologie française. Les mémoires de la Société des Observateurs de l'Homme en l'an VIII, Paris, Le Sycomore, 1978, p. 131. 
21. M. de Certeau, D. Julia, J. Revel, Une politique de la langue. La Révolution française et les patois. L'enquête de Grégoire, 1790-1794, Paris, Gallimard, 1975 ; M.-N. Bourguet, Déchiffrer la France. La statistique départementale à l'époque napoléonienne, Paris, Éd. des Archives Contemporaines, 1988.

22. J. Copans et J. Jamin, Aux origines de l'anthropologie française... ; J. Jamin, « Naissance de l'observation anthropologique : la Société des Observateurs de l'Homme (1799-1805) », Cahiers internationaux de sociologie, 1980. 